\title{
Bücher zum Psychodrama und dessen Umfeld
}

BAMBERGER, GÜNTER, G.: Lösungsorientierte Beratung: Praxishandbuch, Weinheim 2001, 219 Seiten, Euro 24,00.

Buer, Ferdinand (Hrsg.): Praxis der Psychodramatischen Supervision, Leverkusen 2001, 356 Seiten, Euro 30,90.

FABISCH, NiCOLE: Fundraising, München 2002, 352 Seiten, Euro 14,00.

Feltham, Colin und DRYden, Windy: Grundregeln der Supervision, Weinheim-Basel 2002, 132 Seiten, Euro 19,90.

Jensen, Klaus (Hrsg.): Psychotherapie psychodramatisch, Aachen 2001, 186 Seiten.

MARTIN, ERNST: Sozialpädagogische Berufsethik, Weinheim 2001, 264 Seiten, Euro 20,50 .

Maslach, Christina und Leiter, Michael P.: Die Wahrheit über Burnout, Wien 2001, 185 Seiten, Euro 30,40.

MontadA, LeO und Kals, Elesabeth: Mediation, Weinhweim 2001, 301 Seiten, Euro 44,50 .

RitsCheR, WOLFGang: Systemische Modelle für die Soziale Arbeit, Heidelberg 2002, 381 Seiten, Euro 39,90.

Rosenhagen, GÜNTER: Sozialpädagogik und Psychodrama, Frankfurt/M. 2001, Euro 27,60 .

Rost, WOLFGANG: Emotionen. Elexiere des Lebens, Heidelberg 2001, Euro 24,95.

StIMMER, FranZ: Grundlagen des Methodischen Handelns in der Sozialen Arbeit, Stuttgart 2000, 216 Seiten, Euro 20,00.

STIMMER, FranZ (Hrsg.): Lexikon der Sozialpädagogik und der Sozialarbeit, München 2000, (4., völlig überarbeitete und erweiterte Auflage), 813 Seiten.

STIMMER, FRANZ (Hrsg.): Suchtlexikon, München 2000, 645 Seiten.

VOGLaUER, Werner (Hrsg.): Coaching-Praxis, Neuwied 2002, Euro 39,00.

WIENER, Ron: Soziodrama praktisch, München 2001, 77 Seiten.

WitTinger, Thomas (Hrsg.), Psychodrama in der Bildungsarbeit, Mainz 2000, 250 Seiten. 